\title{
WAVE AND STABILITY IN FLUIDS
}


This page is intentionally left blank 


\title{
WAVE AND STABILITY IN FLUIDS
}

\author{
D. Y. Hsieh
}

The Hong Kong University of Science and Technology

S. P. Ho

Hong Kong Polytechnic 


\title{
Published by
}

World Scientific Publishing Co. Pte. Ltd.

P O Box 128, Farrer Road, Singapore 9128

USA office: Suite 1B, 1060 Main Street, River Edge, NJ 07661

UK office: 73 Lynton Mead, Totteridge, London N20 8DH

\author{
Library of Congress Cataloging-in-Publication Data \\ Hsieh, D. Y. (Din-Yu), 1933- \\ Wave and stability in fluids / D.Y. Hsieh, S.P. Ho. \\ p. $\mathrm{cm}$. \\ Includes bibliographical references and index. \\ ISBN 9810218702 \\ 1. Fluid dynamics. 2. Waves. 3. Stability. I. Ho, S. P. \\ II. Title. \\ QC151.H75 1994 \\ 532'.059--dc20 \\ 94-30338 \\ CIP
}

\begin{abstract}
Copyright $\odot 1994$ by World Scientific Publishing Co. Pte. Ltd.
All rights reserved. This book, or parts thereof, may not be reproduced in any form or by any means, electronic or mechanical, including photocopying, recording or any information storage and retrieval system now known or to be invented, without written permission from the Publisher.
\end{abstract}

For photocopying of material in this volume, please pay a copying fee through the Copyright Clearance Center, Inc., 27 Congress Street, Salem, MA 01970, USA.

Printed in Singapore by Uto-Print 


\title{
PREFACE
}

The book is based on the courses given by the first author (DYH) over the years at Brown University, Wuhan University and the Hong Kong University of Science and Technology. It is designed for students in applied mathematics and engineering, who have already been exposed to basic fluid dynamics. The main purpose of the book is to provide relatively easy access to the understanding of the essence of the mechanisms involved in waves and stabilities in fluid. The contents of the book is inevitably influenced by the interests and biases of the authors.

Except for Sections 4.1, 4.2 and 4.3, the writing of the book is essentially carried out by the first author. He has to bear almost all the responsibility of the shortcomings of the book.

The book is largely self-contained. References are given only for supplementation of missing details which cannot be found readily in familiar books such as that listed at the end of the book.

We would like to acknowledge the contributions of graphs in Chapter 6 to our colleague, Dr. Xiao-Ping Wang, and to Mr. Chow Chak On, a second year undergraduate student at HKUST.

Our special thanks to Miss Grace Yeung who typed and produced this camera-ready manuscript, and ingeniously helped us to solve many problem relating to the production of the book.

\author{
D.Y. HSIEH \\ S.P. HO
}

Hong Kong

June 1994 
This page is intentionally left blank 


\section{TABLE OF CONTENTS}

Preface $\quad$ v

1. Preliminaries on Waves 1

1.1 Governing Equations of the Fluid Motion, 2

Eulerian and Lagrangian Coordinates, 6

1.2 The Wave Equation, 8

1.3 Simple Solutions : Sinusoidal Waves, 10

1.4 Fundamental Solutions of Wave Equation, 14

2. Sound Waves

2.1 Waves in Compressible Fluids, 21

2.2 Multi-pole Radiation, 23

2.3 Generation of Sound, 27

2.4 Scattering of Sound, 34

Scattering by a Spherical Bubble in a Liquid, 39

Scattering by a Small Body, 42

2.5 Nonlinear Acoustics, 45

Quadrapole Radiation, 45

Scattering of Sound by Sound, 48

Acoustic Streaming, 53

2.6 Simple Wave and Shock Wave, 56

2.7 Structure of Shock - Burgers Equation, 69 
viii

CONTENTS

Derivation of Burgers Equation, 70

Solution of Burgers Equation, 73

An Example to Illustrate the Shock Structure, 76

2.8 One-Dimensional Nonlinear Wave - Riemann Invariants, 81

2.9 Sound Waves With Thermal Dissipation, 88

\section{Water Waves}

3.1 Governing Equations of Water Waves, 95

3.2 Small Amplitude Waves, 99

Gravity Wave, 103

Capillary Wave, 104

3.3 Dispersion of Waves - Group Velocity, 106

Velocity of Energy Transport, 111

3.4 Wave Produced by a Moving Source, 115

Wave Pattern Due to a Moving Source, 121

3.5 Weakly Nonlinear Waves and Korteweg-DeVries Equation, 129

3.6 Solitary and Cnoidal Waves, 134

3.7 Solitons - Inverse Scattering, 144

3.8 Nonlinear Modulation of Gravity Waves, 153

Stokes Waves, 156

Stability of Stokes Waves, 158

Gravity Waves in Lagrangian Coordinates, 159

3.9 Hydrautic Jumps, 165 
4. Waves in Nonhomogeneous Media

4.1 Internal Waves of Layered Fluid Media, 170 Upper Fluid Surface Free, 176

4.2 Waves in Continuously Stratified Fluids, 179 The Case that $\rho_{0}=\rho^{(0)} e^{-\beta z}, 183$

Rigid Surface on Top, 184

Free Surface on Top, 185

4.3 Waves in Compressible Stratified Fluids, 188

4.4 Dynamic Equations of Bubbly Liquids, 199

A Simple Model of Bubbly Liquids, 201

4.5 Waves in Bubbly Liquids, 202

Sound Waves, 202

Dead Zone and Negative Damping, 209

Waves in Locked Bubbly Liquids, 211

5. Stability

5.1 Interfacial Conditions, 217

5.2 The Classical Rayleigh-Taylor Stability, 223

5.3 Rayleigh-Taylor Stability with Mass and Heat Transfer Across the Interface, 229

A Simplified Version, 236

Nonlinear Stability Based on Simplified Version, 239

5.4 Stability of Spherical Bubble in Motion, 244

Stability of Expanding and Collapsing Bubbles, 252 
5.5 The Classical Kelvin-Helmholtz Stability, 254

5.6 Variational Method and Kelvin-Helmholtz Stability, 262

Nonlinear Analysis, 268

Fully Nonlinear One-Mode Analysis, 271

5.7 Kelvin-Helmholtz Stability for Compressible Fluids, 272

The Case of Semi-infinite Fluid Layers, 277

Relationship with Two Phase Flows, 281

5.8 The Faraday Problem, 285

Linear Stability, 288

Nonlinear Theory, 292

Stability of Oscillating Bubbles, 294

5.9 The Rayleigh-Benard Problem, 296

Principle of Exchange of Stabilities, 304

Critical Rayleigh Number, 307

The Cell Patterns, 310

Non-uniform Heating, 312

5.10 The Taylor-Couette Problem, 313

Rayleigh's Criterion, 316

Linear Stability Analysis, 319

Narrow Gap, 323

5.11 Stability of Parallel Flows, 327

Squire Theorem, 330

Orr-Sommerfeld Equation, 331

Sufficient Conditions for Stability, 332 
Parallel Flow of Inviscid Fluids, 334

Asymptotic Analysis and Comparison

Equations, 337

5.12 Stability of Flow Down an Inclined Plane, 344

Small k Expansion, 349

Hydraulic Approximation, 353

6. Chaos

6.1 The Lorenz Equations, 361

6.2 The Logistic Map, 375

6.3 Characterization of Chaos, 384

Lyapunov Exponents, 384

Dimensions, 387

Dynamical Information in Experimental Data, 390

6.4 Almost Ill-posed Problems and Chaos, 391

Examples of Underlying Ellipticity, 394

Model Partial Differential Equations with Underlying Ellipticity, 398

Appendix $1 \quad$ Some Properties of Spherical Bessel Functions 402

Appendix 2 On Surface Tension

404

Books for Concurrent and Further Reading

References 